\title{
The convective envelope in $\gamma$ Doradus stars: theoretical uncertainties
}

\author{
J. Montalbán, A. Miglio and S. Théado
}

\author{
Institut d'Astrophysique et de Géophysique de I'Université de Liège, B-4000 Liège, Belgium
}

\begin{abstract}
The depth of the convective envelope plays a fundamental role in the driving mechanism proposed by Guzik et al. (2000) to explain the high-order g modes of $\gamma$ Dor pulsators. In this paper we study the sensitivity of the convective envelope depth to the description of convective transport, to relevant physical processes, such as microscopic diffusion, and to other uncertainties in theoretical stellar models.
\end{abstract}

\section{Depth of the convective envelope}

The "convection blocking" of radiation can drive high-order g-modes only for stellar models with a temperature, at the bottom of the convection envelope (CE), between $2 \times 10^{5} \mathrm{~K}$ and $4.8 \times 10^{5} \mathrm{~K}$ (Guzik et al. 2000). Unfortunately, convection modelling is one of the most serious shortcomings in theoretical stellar evolution. The "standard model" of convection, the mixing length theory (MLT), is a simple model that contains essentially one adjustable parameter, $\alpha$, which relates the mixing length to the local pressure scale height. Convection efficiency increases with $\alpha$ as well as, for a given stellar mass and chemical composition, the depth of the CE. Usually $\alpha$ is tuned to produce the solar radius at the solar age, but 2D and 3D numerical simulations of convection suggest that its value should decrease with increasing stellar mass so that, for the $\gamma$ Dor HR domain, it should be lower than the solar value. Furthermore, as the stellar mass increases, the effect of $\alpha$ on the stellar radius decreases, so that $1.5 \mathrm{M}_{\odot}$ stellar models computed with $\alpha$ between 1.8 and 1.4 have the same $T_{\text {eff }}$ (i.e., corresponding to the middle of the observational $\gamma$ Dor instability strip, for a metal mass fraction $Z=0.02$ ) while the depth of their $C E$ is quite different (see Fig.1, left panel).

An alternative to the MLT is the Full Spectrum of Turbulence treatment of convection (FST, Canuto et al. 1996). MLT is more efficient than FST in low efficiency convection regions, while FST is much more efficient than MLT for highly efficient convection. As a consequence, the depth of the CE for FST models changes from shallow to deep in a very narrow domain of $T_{\text {eff }}$ (see Fig. 1, left panel). The range of $T_{\text {eff }}$ of models whose $T_{\mathrm{cz}}$ is between $2 \times 10^{5} \mathrm{~K}$ and $4.8 \times 10^{5} \mathrm{~K}$ is reduced with respect to the MLT case and, therefore, the width of the $\gamma$ Dor instability strip predicted by FST treatment is also smaller.

The depth of the CE for models in the observational $\gamma$ Dor instability strip is also affected by: (1) the microscopic diffusion, that increases by $\mathrm{He}$ settling the $\mathrm{H}$ abundance and, therefore, the opacity in the outer layers (see Fig. 1, right panel), and that, by effect of radiative acceleration and consequent $\mathrm{Fe}$ accumulation, can produce an additional convective region at $2 \times 10^{5} \mathrm{~K}$. (2) the chemical composition: low metallicity models in the instability strip have shallower convective envelopes than solar metallicity ones.

Acknowledgments. The authors acknowledge financial support from the Prodex-ESA Contract Prodex 8 COROT (C90199). 


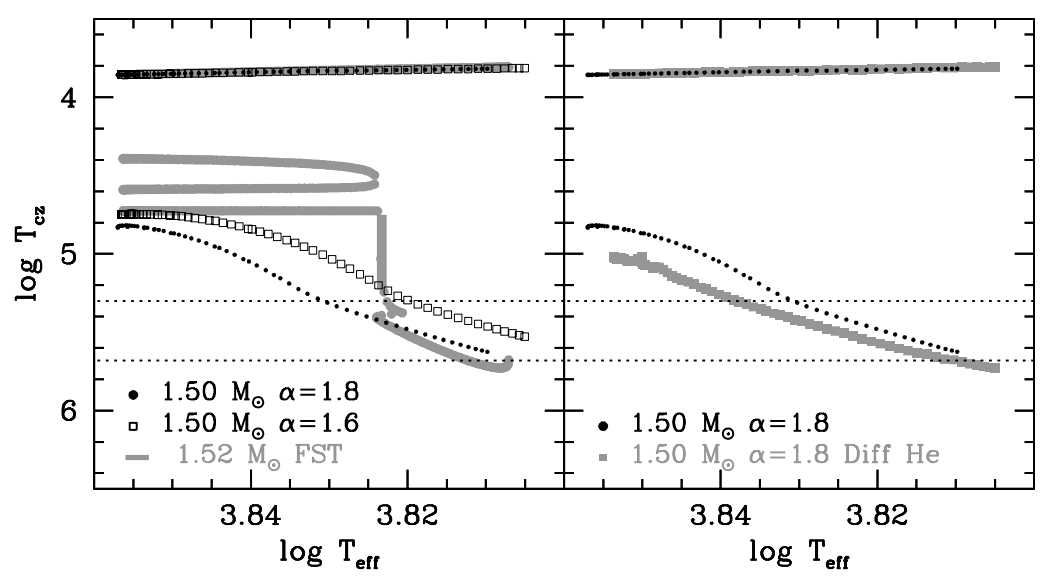

Figure 1: Temperature of the convective envelope boundaries along the main sequence evolution of a $1.5 \mathrm{M}_{\odot}$ star. Left panel: for three different treatments of convection: MLT with $\alpha=1.6$ and 1.8 , and FST. Right panel: models without microscopic diffusion (dots) and with gravitational settling of $\mathrm{He}$ (grey squares).

\section{References}

Guzik J. A., Kaye A. B., Bradley P. A., Cox A. N., Neuforge C., 2000, ApJ, 542, L57

Canuto V. M., Goldman I., Mazzitelli I., 1996, ApJ, 473, 550 